\title{
Fourier Multipliers and Dirac Operators
}

\author{
Craig A. Nolder and Guanghong Wang
}

\begin{abstract}
We use Fourier multipliers of the Dirac operator and Cauchy transform to obtain composition theorems and integral representations. In particular we calculate the multiplier of the $\Pi$-operator. This operator is the hypercomplex version of the Beurling Ahlfors transform in the plane. The hypercomplex Beuling Ahlfors transform is a direct generalization of the Beurling Ahlfors transform and reduces to this operator in the plane. We give an integral representation for iterations of the hypercomplex Beurling Ahlfors transform and we present here a bound for the $L^{p}$-norm. Such $L^{p}$-bounds are essential for applications of the Beurling Ahlfors transformation in the plane. The upper bound presented here is $m\left(p^{*}-1\right)$ where $m$ is the dimension of the Euclidean space on which the functions are defined, $1<p<\infty$ and $p^{*}=\max (p, p /(p-1))$. We use recent estimates on second order Riesz transforms to obtain this result. Using the Fourier multiplier of the $\Pi$ operator we express this operator as a hypercomplex linear combination of second order Riesz transforms.
\end{abstract}

Keywords. Fourier multipliers, Clifford analysis, Dirac operators, П operator, Beurling Ahlfors transform, $L^{p}$-estimates.

msc : $47 \mathrm{~F}, 47 \mathrm{G}, 30 \mathrm{G}$

\section{Introduction}

We determine the Fourier multipliers of the hypercomplex Cauchy transform $T$ and the Vekua $\Pi$-operator over $\mathbb{R}^{n+1}$. Various relations between these operators, and the Dirac and Laplacian operator, are easily obtained using these multipliers. Theorem 3.2 is a convolution identity on $C_{0}^{\infty}\left(\mathbb{R}^{n+1}\right)$ involving the iterated Cauchy kernel and Dirac operator. This is a special case of results in [4]. See also [5] and [6]. Theorem 4.2 shows that a similar convolution integral, with the iterated Cauchy kernel conjugated in this case, represents powers of the $\Pi$-operator. The notation $\Pi$ was used by Vekua [21]. See also [13] and [12]. In the plane this operator has applications to quasiconformal analysis and in that context is called the Beuling-Ahlfors transform [16], [17] . Lemma 4.3 represents the $\Pi$-operator as a hypercomplex combination of second order Riesz transforms. This shows that a hypercomplex combination 
of Riesz transforms is a square root of the $\Pi$-operator. In the plane it is an important open problem to determine the exact value of the $L^{p}$ norms of the Beurling-Ahlfors transform, in particular as an application to two dimensional geometric function theory [16]. Using recent estimates of the $L^{p}$-norms of second order Riesz transforms, obtained in [19], we obtain estimates of the $L^{p}$-norms of the $\Pi$-operator in higher dimensions. In [19] this was done for another higher-dimensional analogue of the Beurling-Ahlfors transform, $S$, to a Grassman algebra. We explore the distinctions between the operators $\Pi$ and $S$.

\section{Preliminaries}

We write $\mathcal{C} \ell_{n}$ for the real universal Clifford algebra over $\mathbb{R}^{n}$. This Clifford algebra is generated as an algebra over $\mathbb{R}$ by the elements

$$
\left\{e_{0}, e_{1}, e_{2}, \ldots, e_{n}\right\} \text {. }
$$

Here $\left\{e_{1}, e_{2}, \ldots, e_{n}\right\}$ is a basis of $\mathbb{R}^{n}$ with the relation $e_{i} e_{j}+e_{j} e_{i}=-2 \delta_{i j}$ and $e_{0}=1$ is the identity and commutes with the basis elements. The dimension of $\mathcal{C} \ell_{n}$ is $\mathbb{R}^{2^{n}}$. We have an increasing tower $\mathbb{R} \subset \mathbb{C} \subset \mathbb{H} \subset \mathcal{C} \ell_{3} \subset \cdots$. Here $\mathbb{H}$ is the quaternions. Using the above relations, products of the elements $\left\{e_{1}, e_{2}, \ldots, e_{n}\right\}$ can be expressed in the form $\pm e_{j_{1}} e_{j_{2}} \cdots e_{j_{k}}$, where $\left\{j_{1}, j_{2}, \ldots, j_{k}\right\} \subset\{1,2, \ldots, n\}$ with $j_{1}<j_{2}<\cdots<j_{k}, 1 \leq k \leq n$. We call the representations $e_{j_{1}} e_{j_{2}} \cdots e_{j_{k}}$ reduced products.

Notice that an element $u \in \mathcal{C} \ell_{n}$ can be written as a linear combination of these reduced products

$$
u=u_{0} e_{0}+\Sigma_{\alpha} u_{\alpha} e_{\alpha}
$$

where the $\alpha$ are the indices of the reduced products, $u_{0}$ and the $u_{\alpha}$ are real.

The Clifford algebra $\mathcal{C} \ell_{n}$ is a graded algebra as $\mathcal{C} \ell_{n}=\oplus_{k=0}^{n} \mathcal{C} \ell_{n}^{k}$ where $\mathcal{C} \ell_{n}^{k}$ are linear combinations of elements whose reduced Clifford products have $k$ basis factors, $k \geq 1$ and $\mathcal{C} \ell_{n}^{0}=\mathbb{R}$. We use the conjugation $\overline{\left(e_{j_{1}} \ldots e_{j_{k}}\right)}=$ $(-1)^{k} e_{j_{k}} \ldots e_{j_{1}}$.

For $A, B \in \mathcal{C} \ell_{n}$ we write $A B$ for the resulting Clifford product. The product $\bar{A} B$ defines a Clifford valued inner product on $\mathcal{C} \ell_{n}$. We have $\overline{A B}=$ $\bar{B} \bar{A}$ and $\overline{\bar{A}}=A$. For $A \in \mathcal{C} \ell_{n}, \operatorname{Sc}(A)$ denotes the scalar part of $A$, that is the coefficient of the element $e_{0}$. The scalar part of a Clifford inner product, $\operatorname{Sc}(\bar{A} B)$, is the usual inner product in $\mathbb{R}^{2^{n}}$ when $A$ and $B$ are identified as vectors. We will denote this usual inner product as $\langle A, B\rangle$.

The space $\mathcal{C} \ell_{n}$ is a Hilbert space with this inner product.

The resulting norm is the usual Euclidean norm given by $\left|u_{0} e_{0}+\Sigma_{\alpha} u_{\alpha} e_{\alpha}\right|=$ $\left(u_{0}^{2}+\Sigma_{\alpha} u_{\alpha}^{2}\right)^{1 / 2}$.

A Clifford valued function $f: \mathbb{R}^{n+1} \rightarrow \mathcal{C} \ell_{n}$ can be written as $f=$ $f_{0} e_{0}+\Sigma_{\alpha} f_{\alpha} e_{\alpha}$ where $f_{0}$ and each $f_{\alpha}$ is real valued and the $e_{\alpha}$ are reduced products. 
We write $\mathcal{C} \ell_{n}^{0,1}$ for the subspace $\mathcal{C} \ell_{n}^{0} \oplus \mathcal{C} \ell_{n}^{1}$. A nonzero element $\zeta \in \mathcal{C} \ell_{n}^{0,1}$ has a multiplicative inverse as $\zeta \bar{\zeta}=|\zeta|^{2}$. We will identify $\mathbb{R}^{n+1}$ with $\mathcal{C} \ell_{n}^{0,1}$ throughout.

We write $L^{p}\left(\mathbb{R}^{n+1}, \mathcal{C} \ell_{n}\right), p>0$, for those functions $f: \mathbb{R}^{n+1} \rightarrow \mathcal{C} \ell_{n}$ such that

$$
\|f\|_{p}=\left(\int_{\mathbb{R}^{n+1}}|f(x)|^{p} d x\right)^{1 / p}
$$

is finite. Here and elsewhere, $d x$ denotes usual Lebesgue measure. We also define the inner product

$$
(f, g)=\int_{\mathbb{R}^{n+1}}\langle f(x), g(x)\rangle d x .
$$

We use the following Cauchy-Riemann operators, with $\left(x_{0}, x_{1}, \ldots, x_{n}\right)$ coordinates of $\mathbb{R}^{n+1}$,

$$
D=\frac{\partial}{\partial x_{0}}+\Sigma_{1}^{n} e_{i} \frac{\partial}{\partial x_{i}}
$$

and

$$
\bar{D}=\frac{\partial}{\partial x_{0}}-\Sigma_{1}^{n} e_{i} \frac{\partial}{\partial x_{i}} .
$$

We have $D \bar{D}=\bar{D} D=\Delta$ where $\Delta$ is the Dirac Laplacian. For $h: \mathbb{R}^{n+1} \rightarrow \mathbb{R}$ we define the Fourier transform

$$
\hat{h}(\zeta)=\int_{\mathbb{R}^{n+1}} h(x) \exp (-i\langle x, \zeta\rangle) d x .
$$

The complex $i$ is not identified with any of the basis elements $e_{1}, e_{2}, \ldots, e_{n}$, commutes with each of them and is invariant under Clifford conjugation, $\bar{i}=i$. Extend the definition linearly

$$
\hat{f}=\hat{f}_{0} e_{0}+\Sigma_{\alpha} \hat{f_{\alpha}} e_{\alpha} .
$$

Identifying $\zeta=\Sigma_{0}^{n} \zeta_{i} e_{i}$ and $\bar{\zeta}=\Sigma_{0}^{n} \zeta_{i} \overline{e_{i}}$,

$$
\widehat{D f}=i \zeta \hat{f}
$$

and

$$
\widehat{\bar{D} f}=i \bar{\zeta} \hat{f}
$$

A given $\zeta$ defines a linear transformation of $\mathcal{C} \ell_{n}$ to itself by the Clifford product $: \omega \rightarrow \zeta \omega$. The matrix in the standard basis (1) is of the form

$$
[\zeta]=\zeta_{0} I+\mathcal{O}
$$

where the matrix $\mathcal{O}$ satisfies

$$
\mathcal{O}^{t}=-\mathcal{O}=\left(|\zeta|^{2}-\zeta_{0}^{2}\right) \mathcal{O}^{-1} .
$$

Furthermore

$$
[\bar{\zeta}]=\zeta_{0} I-\mathcal{O}
$$

It follows that

$$
[i \bar{\zeta}]^{t}=[i \zeta]
$$


corresponding to the roles of $D$ and $\bar{D}$ as adjoint operators. It also follows here that

$$
[i \zeta][i \bar{\zeta}]=-\zeta_{0}^{2} I+\mathcal{O}^{2}=-|\zeta|^{2} I .
$$

This is the matrix for the Laplacian.

For a given convolution operator $A$ with $a \in \mathcal{C} \ell_{n}$ and kernel $k: \mathbb{R}^{n+1} \rightarrow$ $\mathcal{C} \ell_{n}$

$$
A f(x)=a f(x)+\int_{\mathbb{R}^{n+1}} k(x-y) f(y) d y,
$$

we write $\bar{A}$ for the conjugate operator

$$
\bar{A} f(x)=\bar{a} f(x)+\int_{\mathbb{R}^{n+1}} \overline{k(x-y)} f(y) d y .
$$

\section{The Cauchy Transform}

We assume throughout this section that $f \in C_{0}^{\infty}\left(\mathbb{R}^{n+1}, \mathcal{C} \ell_{n}\right)$. We define the Cauchy transform $T$ by

$$
T f(x)=-\frac{1}{\omega} \int_{\mathbb{R}^{n+1}} \frac{\overline{x-y}}{|x-y|^{n+1}} f(y) d y .
$$

Here $\omega$ is the area of the unit sphere in $\mathbb{R}^{n+1}$. It is well known that $T$-operator serves as a right inverse to $D$ and $\bar{T}$ is a right inverse to $\bar{D}$ :

$$
\begin{aligned}
& D T f=f, \\
& \bar{D} \bar{T} f=f .
\end{aligned}
$$

See [12] and [13]. It follows that

$$
\widehat{D T f}=i \zeta \widehat{T f}=\hat{f}
$$

and

$$
\widehat{\bar{D} \bar{T} f}=i \bar{\zeta} \widehat{\bar{T} f}=\hat{f}
$$

Hence the Fourier multiplier of $T$ is $-i \frac{\bar{\zeta}}{|\zeta|^{2}}$, while the Fourier multiplier of $\bar{T}$ is the conjugate $-i \frac{\zeta}{|\zeta|^{2}}$. The multipliers also show that $T$ and $D$ commute on compactly supported functions and that $\bar{T} T$ is $\Delta^{-1}$.

\section{Lemma 3.1.}

$$
\begin{aligned}
& \text { a) }=\bar{T} \bar{D}=I, \\
& \text { b) } T \bar{T} \Delta=\bar{T} T \Delta=I .
\end{aligned}
$$

This identifies $T \bar{T}$ as the Riesz potential of order two,

$$
T \bar{T} f(x)=\Phi_{2} f(x)=\frac{1}{\omega_{n+1}(2-(n+1))} \int_{\mathbb{R}^{n+1}} \frac{f(y)}{|y-x|^{(n+1)-2}} d y,
$$

Hence $\widehat{\Phi_{2} f}=\frac{-1}{|\zeta|^{2}} \hat{f}$. We now define the iterated Cauchy kernel. For $m=$ $1,2, \ldots$, we define the kernel $K_{m}: \mathcal{C} \ell_{n} \rightarrow \mathcal{C} \ell_{n}$ by 


$$
K_{m}(x)=\frac{(-1)^{m} \bar{x}(x+\bar{x})^{m-1}}{\omega_{n} 2^{m-1}(m-1) !|x|^{n+1}} .
$$

We also define the operator

$$
T_{m} f(x)=\int_{\mathbb{R}^{n+1}} K_{m}(y-x) f(y) d y .
$$

When $m=1$ this is the usual Cauchy operator $T=T_{1}$. It follows from direct calculation the for $m=2,3, \ldots$,

$$
D T_{m}=T_{m-1} \text {. }
$$

Because of this we have

$$
\widehat{T_{m} f}=\frac{1}{(i \zeta)^{m}} \hat{f}
$$

and we see that $T_{m}$ is the $m$-fold convolution of $T$ :

$$
T_{m}=T T \cdots T=T \circ T \circ \cdots \circ T
$$

$m$ times. The next result is a special case of results in [4] and [6] and follows from the above multipliers.

Theorem 3.2. For $m=1,2, \ldots$,

$$
f(x)=\int_{\mathbb{R}^{n+1}} K_{m}(y-x) D^{m} f(y) d y .
$$

\section{The Beurling Ahlfors transform}

We define the Hypercomplex Beurling Ahlfors Vekua transform :

$$
\Pi f=\bar{D} T f .
$$

Explicitly

$$
\Pi f(x)=-\frac{1}{\omega} \int_{\mathbb{R}^{n+1}} \frac{(n-1)+(n+1) \frac{\overline{(y-x)}^{2}}{|y-x|^{2}}}{|y-x|^{n+1}} f(y) d y+\frac{1-n}{1+n} f(x) .
$$

When $n=1$ this reduces to the usual Beurling Ahlfors transformation in the plane

$$
\mathcal{B} f(z)=-\frac{1}{\pi} \int_{\mathbb{C}} \frac{f(\zeta)}{(\zeta-z)^{2}} d \zeta
$$

The operator $\Pi$ is bounded on $L^{p}$ and is an isometry on $L^{2}$. See [12]. Using (2) it follows that

$$
\widehat{\Pi f}=i \bar{\zeta} \widehat{T f}=\frac{(\bar{\zeta})^{2}}{|\zeta|^{2}} \hat{f}
$$

As such the multiplier of the Beurling Ahlfors transform is $\frac{(\bar{\zeta})^{2}}{\left.\zeta\right|^{2}}$. When $n=1$ this is the multiplier of the Beurling Ahlfors transform in the plane. Also [12] we have the formula $\bar{\Pi}=D \bar{T}$. It follows from the previous multipliers that the multiplier of $\bar{\Pi}$ is $\frac{\zeta^{2}}{|\zeta|^{2}}$, which is the conjugate the multiplier of $\Pi$. This 
also follows from the Hecke identities, see [20] and [16]. The above multipliers give the following results.

Lemma 4.1. On $C_{0}^{\infty}\left(\mathbb{R}^{n+1}, \mathcal{C} \ell_{n}\right)$,
a)
$\Pi \bar{\Pi}=\bar{\Pi} \Pi=I$,
b)
$\Pi D=\bar{D}$,
c)
$D \Pi=\bar{D}$,
d)
$\Pi=\bar{D}^{2} \Delta^{-1}$.

See [12] and [13]. The properties Lemma $4.1 b)$ and $c$ ) are useful to solve hypercomplex Beltrami equations of the form $D f=\mu(x) \bar{D} f$.

The following result parallels Theorem 3.2. See [8] and [7].

Theorem 4.2. Assume that $n>1$ and $f \in C_{0}^{\infty}\left(\mathbb{R}^{n+1}, \mathcal{C} \ell_{n}\right)$. For $m=1,2, \ldots$,

$$
\Pi^{m} f(x)=\int_{\mathbb{R}^{n+1}} K_{m}(y-x) \bar{D}^{m} f(y) d y=\int_{\mathbb{R}^{n+1}} \overline{K_{m}(y-x)} D^{m} f(y) d y .
$$

The Riesz transforms are defined, for $j=0, \ldots, n$, as follows

$$
R_{j} f(x)=-\frac{1}{\omega} \int_{\mathbb{R}^{n+1}} \frac{x_{j}-y_{j}}{|x-y|^{n+2}} f(y) d y .
$$

It is well known (and follows from the Hecke identities [20],[17]) that

$$
\widehat{R_{j} f}=\frac{i \zeta_{j}}{|\zeta|} \hat{f}
$$

We also define the Clifford Riesz transforms as

$$
\mathcal{R}=R_{0}+\Sigma_{j=1}^{n} R_{j} e_{j}
$$

and

$$
\overline{\mathcal{R}}=R_{0}-\Sigma_{j=1}^{n} R_{j} e_{j} .
$$

Since $\frac{\bar{\zeta}}{|\zeta|}$ is the square root of the multiplier for $\Pi, \mathcal{H}=\frac{1}{i} \overline{\mathcal{R}}$ is the square root of $\Pi$ and so

$$
\Pi=\mathcal{H} \mathcal{H}=-\overline{\mathcal{R}} \overline{\mathcal{R}}
$$

We remark that

$$
\mathcal{H} \overline{\mathcal{H}}=-\mathcal{R} \overline{\mathcal{R}}=-R_{0}{ }^{2}-\Sigma_{j=1}^{n} R_{j}{ }^{2}=I .
$$

Hence $\mathcal{H}$ serves as a Clifford valued Hilbert transform. Furthermore the operator $\Pi$ can be expressed in terms of second order Riesz transforms.

\section{Lemma 4.3 .}

$$
\Pi=-R_{0}^{2}+\Sigma_{j=1}^{n} R_{j}^{2}+2 \Sigma_{j=1}^{n} R_{0} R_{j} e_{j}
$$


The matrix form of the multiplier of $\Pi$ is then given by

$$
\frac{2 \zeta_{0}^{2}-|\zeta|^{2}}{|\zeta|^{2}} I+\frac{2 \zeta_{0}}{|\zeta|^{2}} \mathcal{O}
$$

while that of $\bar{\Pi}$ is

$$
\frac{2 \zeta_{0}^{2}-|\zeta|^{2}}{|\zeta|^{2}} I-\frac{2 \zeta_{0}}{|\zeta|^{2}} \mathcal{O} .
$$

In the complex case, $n=1$, the matrix $\mathcal{O}$ is of the form

$$
\left(\begin{array}{cc}
0 & -\zeta_{1} \\
\zeta_{1} & 0
\end{array}\right)
$$

and the matrix of the multiplier of $\Pi$ is

$$
\frac{1}{|\zeta|^{2}}\left(\begin{array}{cc}
\zeta_{0}^{2}-\zeta_{1}^{2} & -2 \zeta_{0} \zeta_{1} \\
2 \zeta_{0} \zeta_{1} & \zeta_{0}^{2}-\zeta_{1}^{2}
\end{array}\right) .
$$

In the case of the quaternions, $n=2$, the matrix $\mathcal{O}$ is of the form

$$
\left(\begin{array}{cccc}
0 & -\zeta_{1} & -\zeta_{2} & 0 \\
\zeta_{1} & 0 & 0 & \zeta_{2} \\
\zeta_{2} & 0 & 0 & -\zeta_{1} \\
0 & -\zeta_{2} & \zeta_{1} & 0
\end{array}\right)
$$

Hence the matrix of the multiplier of $\Pi$ in this case is

$$
\frac{1}{|\zeta|^{2}}\left(\begin{array}{cccc}
2 \zeta_{0}^{2}-|\zeta|^{2} & -2 \zeta_{0} \zeta_{1} & -2 \zeta_{0} \zeta_{2} & 0 \\
2 \zeta_{0} \zeta_{1} & 2 \zeta_{0}^{2}-|\zeta|^{2} & 0 & 2 \zeta_{0} \zeta_{2} \\
2 \zeta_{0} \zeta_{2} & 0 & 2 \zeta_{0}^{2}-|\zeta|^{2} & -2 \zeta_{0} \zeta_{1} \\
0 & -2 \zeta_{0} \zeta_{2} & 2 \zeta_{0} \zeta_{1} & 2 \zeta_{0}^{2}-|\zeta|^{2}
\end{array}\right) .
$$

The complex Beurling Ahlfors transform has also been generalized to the Grassman algebra, [16], [17], by the following definition :

$$
S=(d \delta-\delta d) \Delta^{-1}: \Lambda_{n+1}^{k} \rightarrow \Lambda_{n+1}^{k}
$$

Here $\Lambda_{n+1}=\oplus_{k} \Lambda_{n+1}^{k}$ is the graded Grassman algebra over $\mathbb{R}^{n+1}$, generated by $d x_{0}, d x_{1}, \ldots, d x_{n}$, with exterior derivative $d$ and formal Hodge adjoint $\delta$. Notice the similarities with the formula in Lemma $4.1 \mathrm{~d}$ ).

Although $S$ is also expressed in terms of second order Riesz transforms the internal structures of $\Pi$ and $S$ are quite different. First the operator $S$ preserves the grading of the Grassman algebra where as $\Pi: \mathcal{C} \ell_{n}^{k} \rightarrow \mathcal{C} \ell_{n}^{k+2} \oplus$ $\mathcal{C} \ell_{n}^{k} \oplus \mathcal{C} \ell_{n}^{k-2}$. Because of this, unlike $\Pi, S$ has a block diagonal form : $S=$ $\operatorname{diag}\left(1, S_{1}, \ldots, S_{n},-1\right)$ where $S_{k}: \Lambda_{n+1}^{k} \rightarrow \Lambda_{n+1}^{k}$. In the plane, $n=1$, the matrix of $\Pi$ has the form

$$
\Pi=\left(\begin{array}{cc}
R_{1}^{2}-R_{0}^{2} & -2 R_{0} R_{1} \\
2 R_{0} R_{1} & R_{1}^{2}-R_{0}^{2}
\end{array}\right) .
$$

This is identical with the Beurling-Ahlfors transform. In comparison,

$$
S=\left(\begin{array}{ccc}
1 & 0 & 0 \\
0 & S_{1} & 0 \\
0 & 0 & -1
\end{array}\right)
$$


where

$$
S_{1}=\left(\begin{array}{cc}
R_{0}^{2}-R_{1}^{2} & -2 R_{0} R_{1} \\
-2 R_{0} R_{1} & R_{1}^{2}-R_{0}^{2}
\end{array}\right) .
$$

Hence $S_{1}$ can be identified with $\Pi$ under a linear change of variables in the case that $n=1$. In higher dimensions the matrix of $\Pi$ is $2^{n} \times 2^{n}$. In comparison the matrix of $S$ is $2^{n+1} \times 2^{n+1}$ and the $k$ th blocks have dimensions $\frac{(n+1) !}{(n+1-k) ! k !} \times \frac{(n+1) !}{(n+1-k) ! k !}$. Moreover the diagonal entries of $\Pi$ are identical, always having the form in the matrix (13). In comparison the diagonal entries of $S$ have minus signs that are permuted across the sums. See [16]. Because of these observations the operators do not directly compare in dimensions $n+1, n \geq 2$.

Next we give an estimate of the $L^{p}$-norm of the $\Pi$ operator. For the operator $S$ a similar result appears in [19]. The inequalities follow by separately estimating the norms of the second order Riesz transforms. It is suspected that the true values of these norms will be revealed from a study of the internal structure of these operators and the averaging properties which they possess. See the work [14].

Theorem 4.4. The $L^{p}$-norm of $\Pi: L^{p}\left(\mathbb{R}^{n+1}, \mathcal{C} \ell_{n}\right) \rightarrow L^{p}\left(\mathbb{R}^{n+1}, \mathcal{C} \ell_{n}\right), 1<p<$ $\infty$, has the estimate

$$
\|\Pi\|_{p} \leq(n+1)\left(p^{*}-1\right) .
$$

Here $p^{*}=\max (p, p /(p-1))$.

In 1983 Iwaniec proposed the following conjecture [15],

Conjecture 4.5. When $n=1$ the complex Beurling Ahlfors transform has $L^{p}$-norm, $1<p<\infty$,

$$
\|\mathcal{B}\|_{p}=p^{*}-1 \text {. }
$$

Unfortunately, Theorem 4.4 does not attain the conjectured constant in the plane, reducing to 2 instead of 1 . As such the bound is far away from the Iwaniec conjecture. In the plane the constant 2 was obtained in [18] using a special Bellman function. See also [9], [10] and [11].

Using martingale transforms the constant 2 is obtained in [3] and is reduced to 1.575 in [1]. Martingale techniques have been used subsequently to improve $L^{p}$-bounds for the Grassmann operator, $S$, see [2].

Although often results in complex analysis in the plane do not extend to hypercomplex analysis, the algebraic similarities of the Fourier multipliers suggest the following conjecture.

Conjecture 4.6. The norm of the hypercomplex Beurling Ahlfors transform $\Pi: L^{p}\left(\mathbb{R}^{n+1}, \mathcal{C} \ell_{n}\right) \rightarrow L^{p}\left(\mathbb{R}^{n+1}, \mathcal{C} \ell_{n}\right), 1<p<\infty$, is

$$
\|\Pi\|_{p}=p^{*}-1
$$

in all dimensions. 
This conjecture for the $S$-operator appears in [16].

\section{Proof of Theorem 4.4}

We include an outline of the proof of Theorem 4.4 here for completeness. Theorems 5.1 and 5.2 are proved in [19]. Below $\mathcal{E}$ denotes a Hilbert space with inner product $\langle,\rangle_{\mathcal{E}}$ and corresponding norm $|\cdot|_{\mathcal{E}}$. Here $R_{l}, l=0, \ldots, n$ are the Riesz transforms as above, $\phi, \psi: \mathbb{R}^{n+1} \rightarrow \mathcal{E}$ are test functions and $\tilde{\phi}, \tilde{\psi}$ are extensions of $\phi$ and $\psi$ to $\mathbb{R}_{+}^{n+2}$. These extensions are solutions to the heat equation with $\phi$ and $\psi$ as initial data.

Theorem 5.1. For $j, k=0, \ldots, n$.

$$
\int_{\mathbb{R}^{n+1}}\left\langle R_{j} R_{k} \phi, \psi\right\rangle_{\mathcal{E}} d x=-2 \int_{\mathbb{R}_{+}^{n+2}}\left\langle\frac{\partial}{\partial x_{j}} \tilde{\phi}, \frac{\partial}{\partial x_{k}} \tilde{\psi}\right\rangle_{\mathcal{E}} d x d t
$$

Theorem 5.2. For $1 / p+1 / q=1$,

$$
\begin{gathered}
2 \sum_{i, j=0}^{n} \int_{\mathbb{R}_{+}^{n+2}}\left|\frac{\partial}{\partial x_{i}} \tilde{\phi}\right|_{\mathcal{E}}\left|\frac{\partial}{\partial x_{j}} \tilde{\psi}\right|_{\mathcal{E}} d x d t \\
\leq(n+1)(p-1)\|\phi\|_{L^{p}\left(\mathbb{R}^{n+1}, \mathcal{E}\right)}\|\psi\|_{L^{q}\left(\mathbb{R}^{n+1}, \mathcal{E}\right)} .
\end{gathered}
$$

Using the representation of Lemma 4.3, Theorem 5.1 and Theorem 5.2 with $\mathcal{E}=\mathcal{C} \ell_{n}$ and Hölder's inequality we obtain

$$
\begin{gathered}
|(\Pi \phi, \psi)|=\left|\int_{\mathbb{R}^{n+1}}\langle\Pi \phi, \psi\rangle\right| \\
\leq\left|\int_{\mathbb{R}^{n+1}}\left\langle R_{0}^{2} \phi, \psi\right\rangle\right|+\sum_{j=1}^{n}\left|\int_{\mathbb{R}^{n+1}}\left\langle R_{j}^{2} \phi, \psi\right\rangle\right| \\
+\sum_{j=1}^{n}\left|\int_{\mathbb{R}^{n+1}}\left\langle R_{0} R_{j} \phi, \psi\right\rangle\right|+\sum_{j=1}^{n}\left|\int_{\mathbb{R}^{n+1}}\left\langle R_{j} R_{0} \phi, \psi\right\rangle\right| \\
=2\left|\int_{\mathbb{R}_{+}^{n+2}}\left\langle\frac{\partial}{\partial x_{0}} \tilde{\phi}, \frac{\partial}{\partial x_{0}} \tilde{\psi}\right\rangle\right|+2 \Sigma_{j=1}^{n}\left|\int_{\mathbb{R}_{+}^{n+2}}\left\langle\frac{\partial}{\partial x_{j}} \tilde{\phi}, \frac{\partial}{\partial x_{j}} \tilde{\psi}\right\rangle\right| \\
+2 \Sigma_{j=1}^{n}\left|\int_{\mathbb{R}_{+}^{n+2}}\left\langle\frac{\partial}{\partial x_{0}} \tilde{\phi}, \frac{\partial}{\partial x_{j}} \tilde{\psi}\right\rangle\right| \\
+2 \Sigma_{j=1}^{n}\left|\int_{\mathbb{R}_{+}^{n+2}}\left\langle\frac{\partial}{\partial x_{j}} \tilde{\phi}, \frac{\partial}{\partial x_{0}} \tilde{\psi}\right\rangle\right| \leq 2 \Sigma_{i, j=0}^{n} \int_{\mathbb{R}_{+}^{n+2}}\left|\frac{\partial}{\partial x_{i}} \tilde{\phi} \| \frac{\partial}{\partial x_{j}} \tilde{\psi}\right| \\
\leq(n+1)(p-1)\|\phi\|_{p}\|\psi\|_{q} .
\end{gathered}
$$

With the choice $\psi=\Pi \phi|\Pi \phi|^{(p / q)-1}$ and using density and duality, we obtain Theorem 4.4. 


\section{References}

[1] R. Banuelos and P. Janakiraman, $L^{p}$-bounds for the Beurling-Ahlfors transform, Trans. Amer. Math. Soc., 360: 3603-3612, 2008.

[2] R. Banuelos and A. Linderman II, A martingale study of the Beurling-Ahlfors transform in $\mathbb{R}^{n}$, J. Funct. Anal., 145(1): 224-265, 1997.

[3] R. Banuelos and P. J. Mendez-Hernandez, Sharp inequalities for the heat kernels of Schrödinger operators and applications to spectral gaps, J. Funct. Anal., 176(2):368-399, 2000.

[4] H. Begehr, Iterated Integral Operators in Clifford Analysis, Journal for Analysis and Applications, 18 (2) : 361-377, 1999.

[5] H. Begehr, Integral representations in complex hypercomplex and Clifford analysis, Integral Transforms and Special Functions, 13 : 223-241.

[6] H. Begehr and J. Dubinskii, Orthogonal decompositions of Sobolev spaces in Clifford analysis, Annali di Matematica, 181 : 55-71, 2002.

[7] P. Cerejeiras, K. Gürlebeck, U. Käler and H. Malonek, A quternionic Beltramitype equation and the existince of local homeomorphic solutions, Journal for Analysis and Applcations, 20(1): 17-34, 2001.

[8] P. Cerejeiras and U. Käler, On Beltrami equations in Clifford analysis and its quasiconformal solutions, Proceedings of the NATO Advanced Research Workshop on Clifford Analysis and its Applications, Prague, Czech Republic, 2000.

[9] O. Dragicevic and A. Volberg, Bellman functions and dimensionless estimates of the Littlewood-Paley type, J. Operator Theory, 56(4): 167-198, 2006.

[10] O. Dragicevic and A. Volberg, Bellman function, Littlewood-Paley estimates and asymtotics for the Ahlfors-Beurling operator in $L^{p}(\mathbb{C})$, Indiana Univ. Math. J. , 54(4): 971-995, 2005.

[11] O. Dragicevic and A. Volberg, Bellman function for the estimate of LittlewoodPaley type and asymtotic estimates in the $p-1$ problem, C. R. Math. Acad. Sci. Paris, 340(10): 731-734, 2005.

[12] K. Gürlebeck and U. Kähler, On a spatial generalization of the complex Пoperator, Journal for Analysis and its Applications, 15(2): 283-297, 1996.

[13] K. Gürlebeck, On some classes of П-operators, Dirac operators in analysis, Addison Wesley Longman Limited, 1998.

[14] A. Hinkkanen, On the norm of the Beurling Ahlfors transform, preprint.

[15] T. Iwaniec, Extremal inequalities in Sobolev spaces and quasiconformal mappings, Z. Anal. Anwendungen, 1(6): 1-16, 1982.

[16] T. Iwaniec and G. Martin, Geometric Function Theory and Non-linear Analysis, Oxford Mathematical Monographs, Oxford University Press, New York, 2001.

[17] T. Iwaniec and G. Martin, Riesz transforms and related singular integrals, $J$. Reine Angew. Math., 473: 25-57, 1996.

[18] F. Nazarov and A. Volberg, Heat extension of the Beurling operator and estimates of its norm, Algebra i Analiz, 15(4): 563-573, 2004.

[19] S. Petermichl, L. Slavin and B. Wick, New estimates for the Beurling-Ahlfors operator on differential forms, to appear.

[20] E.M. Stein, Singular Integrals and Differentiability Properties of Functions, Princeton University Press, 1970. 
[21] I. N. Vekua, Generalized Analytic Functions, Pergamon Press, Oxford, 1962.

Craig A. Nolder and Guanghong Wang 